\title{
Clinical Safety and Effectiveness of Stent-Assisted Coil Embolization with Neuroform Atlas Stent in Intracranial Aneurysm
}

\author{
Chang Hyeun Kim, Young Ha Kim, Soon Ki Sung, Dong Wuk Son, Geun Sung Song, Sang Weon Lee \\ Department of Neurosurgery, Pusan National University Yangsan Hospital, Pusan National University School of Medicine, Yangsan, Korea
}

Objective : Stent-assisted coil embolization (SAC) is commonly used for treating wide-neck intracranial aneurysms. In this study, we aimed to assess the clinical safety and efficacy of the NeuroForm Atlas Stent during SAC of intracranial aneurysms.

Methods : We retrospectively analyzed data from patients with ruptured and unruptured cerebral aneurysms, who underwent SAC using the NeuroForm Atlas between February 2018 and July 2018. Favorable clinical outcomes and degree of aneurysm occlusion were defined as a modified Rankin scale score of $\leq 2$ and a Raymond-Roy occlusion classification (RROC) class I/II during the immediate postoperative period and at the 6-month follow-up, respectively.

Results : Thirty-one consecutive patients with 33 cases, including 11 ruptured and 22 unruptured cases were treated via NeuroForm Atlas SAC. Among the 22 unruptured cases with 24 unruptured aneurysms had favorable clinical outcome. Complete occlusion (RROC I) was achieved in 16 aneurysms (66.7\%), while neck remnants (RROC II) were observed in six aneurysms (25\%). Among the 11 patients with ruptured aneurysms, two died due to re-bleeding and diabetic ketoacidosis. In ruptured cases, RROC I was observed in eight (72.7\%) and RROC II was observed in three cases (27.3\%). At the 6-month follow-up, no clinical events were observed in the 22 unruptured cases. In the ruptured nine cases, five patients recovered without neurologic deficits, while four experienced unfavorable outcomes at 6 months. Of the 29 aneurysms examined via angiography at the 6-month follow-up, 19 (65.5\%) were RROC I, eight (27.6\%) were RROC II and two (6.9\%) were RROC III. There were no procedure-related hemorrhagic complications.

Conclusion : In this study, we found that stent-assisted coil embolization with NeuroForm Atlas stent may be safe and effective in the treatment of wide-neck intracranial aneurysms. NeuroForm Atlas SAC is feasible for the treatment of both ruptured and unruptured wide-neck aneurysms.

Key Words : Intracranial aneurysm · Stents · Coil.

- Received : July 3, 2019 •Revised : August 15, 2019 •Accepted : October 10, 2019

- Address for reprints : Sang Weon Lee

Department of Neurosurgery, Pusan National University Yangsan Hospital, 20 Geumo-ro, Mulgeum-eup, Yangsan 50612, Korea

Tel : +82-55-360-2126, Fax : +82-55-360-2156, E-mail : sangweonlee@pusan.ac.kr, ORCID : https://orcid.org/0000-0002-3199-7072

This is an Open Access article distributed under the terms of the Creative Commons Attribution Non-Commercial License (http://creativecommons.org/licenses/by-nc/4.0) which permits unrestricted non-commercial use, distribution, and reproduction in any medium, provided the original work is properly cited. 


\section{INTRODUCTION}

Endovascular treatment of intracranial aneurysms using coils has become an accepted alternative to aneurysm neck clipping $^{3,4,8)}$. Technological advancements have led to increased coil stability, improved microwire stability and flexibility, and development of smaller microcatheters, which have rendered the process safer and simpler. However, incomplete occlusion or coil protrusion can occur during endovascular coiling, especially in patients with wide-neck or complex aneurysms. Stent-assisted coil embolization is being commonly used to counter such problems in the treatment of wide-neck aneurysms.

In the past 20 years, several newer laser-cut or braded stents have been introduced, such as the Solitaire, NeuroForm EZ, Enterprise, LEO and LEO Baby, LVIS and LVIS Jr. stent. Each stent has its own advantages and disadvantages. The open-cell type stents have the advantage of a better apposition of the vessel wall compared to that with the closed-cell type stents, but re-sheathing is impossible, and coil prolapse is more frequent in patients with open-cell stents ${ }^{7}$. Braded stents have increased metal coverage and can expect the flow diversion effects; however, considerable effort is required for the proper deployment ${ }^{5)}$. NeuroForm Atlas (Stryker Neurovascular, Fremont, CA, USA) microstent is a next generation stent that has been improved to ensure lower profile delivery (via 0.0165 inch inner diameter microcatheter), better scaffolding due to small cell sizes, improved trackability, and higher conformability to the vessel wall compared to that with the previous stents. However, not many reports of this NeuroForm Atlas stent have aimed to evaluate the safety and efficacy of the NeuroForm Atlas during stent-assisted coil embolization of intracranial aneurysms.

\section{MATERIALS AND METHODS}

\section{Patient population}

We retrospectively analyzed electronic medical records and picture archiving and communication system data from 31 consecutive patients with intracranial aneurysms treated at our institution using the NeuroForm Atlas (Stryker Neurovascular) stent-assisted coil embolizaiton (SAC) between February 2018 and July 2018. Patients with both ruptured and unruptured aneurysms were included. This study was approved by Institutional Review Board of Pusan National University Yangsan Hospital (IRB No. 05-2019-090).

In addition to aneurysm-related information (location, size, prior treatment, and dome-neck ratio), we collected detailed procedural information regarding the techniques used (i.e., jailing or trans-strut technique), angiographic outcomes (Raymond-Roy occlusion classification, RROC), and periprocedural complications. Follow-up clinical and angiographic data were also obtained. In-stent stenosis was defined as narrowing of the vessel by more than 10\%. Angiographic results were independently adjudicated by two neurovascular specialists.

\section{Endovascular procedural information}

Patients with unruptured aneurysms were pre-medicated using dual antiplatelet medication (acetylsalicylic acid $100 \mathrm{mg}$ daily and clopidogrel $75 \mathrm{mg}$ daily) for at least 7 days. P2Y12 reaction units were analyzed using the Verify Now assay prior to the procedure, and patients with values greater than 240 were regarded as clopidogrel non-responders. In such patients, cilostazol was administered immediately before the procedure, and clopidogrel was replaced with cilostazol after the procedure. During the procedure, 3000 IU of heparin was administered (depending on the patient's bodyweight, $50 \mathrm{IU} / \mathrm{kg}$ ). If the procedural time exceeded 1 hour, additional 1000 IU of heparin were administered per hour.

The preoperative dual antiplatelet medication was not indicated in ruptured cases. Therefore, glycoprotein IIb/IIIa inhibitor tirofiban (Aggrastat, Merck \&Co, Kenilworth, NJ, USA) was administered intravenously after stent deployment. All such patients received maintenance infusions of tirofiban $(0.1 \mu \mathrm{g} / \mathrm{kg} / \mathrm{min} \text { intravenously for } 24 \text { hours })^{13)}$.

All procedures were performed under general anesthesia and via femoral artery access with systemic heparinization. The flush system contained 5000 IU of heparin per liter.

\section{Measurement of Clinical and angiographic out- come}

Clinical outcomes were evaluated by the neurosurgeon using the modified Rankin scale (mRS). According to the mRS, favorable and unfavorable outcomes were 0-2 and 3-6, respectively. In patients with ruptured aneurysms, the HuntHess grades, Fisher's grades, Glasgow coma scale scores, and the $\mathrm{mRS}$ scores were recorded at initial evaluation, while 
changes in mRS scores were recorded at discharge and during outpatient follow-up.

Digital subtraction angiography (DSA) was performed at the 6-month follow-up appointment. Occlusion of the aneurysm was evaluated based on the guidelines of the RROC (class I : complete obliteration, class II : residual neck, class III : residual sac). Recanalization was defined as a change from RROC class I/II to class III between the immediate postoperative period and the end of follow-up. All changes, including coil configuration, flow patency of the parent artery, and intimal hyperplasia, were recorded.

\section{Safety and efficacy}

Occurrence of major ipsilateral stroke or neurological death between the immediate postoperative period and 6 months following the procedure was considered as the primary endpoint. We compared our safety data with long-term morbidity and mortality data derived from previous studies evaluating

Table 1. Unruptured aneurysm features and details of the angiographic procedure

\begin{tabular}{|c|c|c|c|c|c|c|c|c|c|c|}
\hline No. & Location & $\begin{array}{l}\text { Parent } \\
\text { vessel } \\
\text { diameter } \\
\text { proximal } \\
(\mathrm{mm})\end{array}$ & $\begin{array}{c}\text { Parent } \\
\text { vessel } \\
\text { diameter } \\
\text { distal } \\
(\mathrm{mm})\end{array}$ & $\begin{array}{c}\text { Dome/neck } \\
(\mathrm{mm})\end{array}$ & Treatment & $\begin{array}{l}\text { Stent } \\
\text { size }\end{array}$ & RROC & $\begin{array}{c}\text { FU RROC } \\
\text { at } 6 \\
\text { months }\end{array}$ & $\begin{array}{l}\text { Clinical } \\
\text { outcomes } \\
\text { BP/AP }\end{array}$ & Etc. \\
\hline 1 & MCAB & 2.7 & 1.8 & $2.5 / 3.6$ & j & $3.0 \times 15$ & 1 & 1 & $0 / 0$ & \\
\hline 2 & SHP & 4.9 & 4.7 & $3.5 / 4.5$ & j & $4.5 \times 21$ & 1 & 1 & $0 / 0$ & \\
\hline 3 & BA & 3.2 & 2.0 & $4.2 / 4.1$ & $j, s$ & $3.0 \times 15$ & 2 & 2 & $0 / 0$ & \\
\hline 4 & SHP & 4.6 & 4.2 & $6.0 / 4.3$ & $d, s$ & $4.5 \times 21$ & 1 & 2 & $0 / 0$ & \\
\hline 5 & Paraclinoid & 6.3 & 4.4 & $6.0 / 3.0$ & j & $4.5 \times 21$ & 1 & 1 & $0 / 0$ & Coil protrusion \\
\hline 6 & SHP/paraophthalmic & 4.7 & 4.2 & $4.2 / 4.0,2.5 / 2.8$ & $d, j$ & $4.5 \times 21$ & $1 / 2$ & $1 / 2$ & $0 / 0$ & \\
\hline 7 & SCA & 4.2 & 2.5 & $2.4 / 2.6$ & j & $3.0 \times 21$ & 1 & 1 & $0 / 0$ & \\
\hline 8 & PCOA/SHP & 5.3 & 4.8 & $\begin{array}{l}3.8 / 4.3 \\
4.1 / 3.2\end{array}$ & $d, j$ & $4.5 \times 21$ & $1 / 2$ & $1 / 2$ & $0 / 0$ & \\
\hline 9 & $B A$ & 3.3 & 3.1 & $2.5 / 2.1$ & j & $3.0 \times 15$ & 1 & 1 & $0 / 0$ & \\
\hline 10 & Paraophthalmic & 4.5 & 4.2 & $3.6 / 3.6$ & j & $4.5 \times 21$ & 2 & 2 & $0 / 0$ & \\
\hline 11 & $\mathrm{ACOA}$ & 4.2 & 2.8 & $6.0 / 5.5$ & $d, j$ & $4.0 \times 21$ & 2 & 2 & $0 / 0$ & \\
\hline 12 & PCoA & 4.9 & 4.4 & $3.6 / 4.5$ & j & $4.5 \times 21$ & 1 & 1 & $0 / 0$ & \\
\hline 13 & ICA dorsal wall & 4.6 & 4.4 & $2.7 / 3.4$ & j & $4.5 \times 21$ & 1 & & $0 / 0$ & \\
\hline 14 & cavernous & 4.9 & 4.6 & $3.6 / 4.5$ & j & $4.5 \times 21$ & 3 & 2 & $0 / 0$ & \\
\hline 15 & SHP & 4.3 & 3.8 & $4.0 / 3.0$ & j.s & $4.5 \times 21$ & 2 & 1 & $0 / 0$ & \\
\hline 16 & ICA dorsal wall & 4.7 & 3.0 & & j & $4.5 \times 21$ & 1 & 1 & $2 / 2$ & $\begin{array}{l}\text { SAH (case No. 33), } \\
\text { recoiling }\end{array}$ \\
\hline 17 & BA & 3.3 & 2.5 & $3.6 / 5.3$ & $j, s$ & $3.0 \times 15$ & 1 & 1 & 0/0 & $\begin{array}{l}\text { Same patient } \\
\text { case No. } 18\end{array}$ \\
\hline 18 & SHP & 4.8 & 4.1 & $4.0 / 3.0$ & j,s & $4.5 \times 21$ & 1 & 1 & $0 / 0$ & $\begin{array}{l}\text { Same patient } \\
\text { case No. } 17\end{array}$ \\
\hline 19 & $\mathrm{ACOA}$ & 3.3 & 3.0 & $2.8 / 3.8$ & j & $3.0 \times 21$ & 1 & 2 & $0 / 0$ & \\
\hline 20 & $\mathrm{BA}$ & 3.3 & 2.6 & $2.5 / 2.5$ & j & $4.5 \times 21$ & 3 & 3 & $0 / 0$ & \\
\hline 21 & Paraclinoid & 5.5 & 5.1 & $2.2 / 2.5$ & j & $3.0 \times 21$ & 1 & 1 & $0 / 0$ & \\
\hline 22 & $\mathrm{ACOA}$ & 2.4 & 2.3 & & j & $3.0 \times 21$ & 1 & 1 & $0 / 0$ & $\begin{array}{l}\text { Previous SAH, } \\
\text { recoiling }\end{array}$ \\
\hline
\end{tabular}

RROC : Raymond-Roy occlusion classification, FU : follow-up, BP : before procedure, AP : after procedure, MCAB : middle cerebral artery bifurcation, $\mathrm{j}$ : jailing technique, SHP : superior hypophyseal artery, BA : Basilar artery, s: trans-strut technique, $d$ : double catheter technique, SCA : superior cerebellar artery, PCOA : posterior communicating artery, ACOA : anterior communicating artery, ICA : internal carotid artery, SAH : subarachnoid hemorrhage 
stent-assisted coil embolization. All procedural events relating to the device, including coil loop protrusion, thromboembolic events, re-bleeding, or changes in technique, were recorded and evaluated. Angiographic results (RROC classes I and II) at the 6-month follow-up appointment and rate of aneurysm occlusion (complete occlusion) were regarded as the primary efficacy endpoints.

\section{RESULTS}

\section{Characteristics of patients and aneurysms}

Thirty-one consecutive patients with 35 aneurysms underwent 33 stent-assisted coil embolization procedures using NeuroForm Atlas between February 2018 and July 2018. In two patients, aneurysms were located close to one another, allowing simultaneous treatment using a single stent-assisted embolization. In one patient, two aneurysms were located far from one another, necessitating two separate stent-assisted embolization procedures. In one patient, two stents were used for one aneurysm. All patients were included in the analysis. Among the 31 treated patients, 11 (35.5\%) were treated for ruptured aneurysms, while 21 were treated for unruptured aneurysms, including one patient who underwent retreatment. The mean patient age was $60.6 \pm 13.3$ years (range, $22-$ 83), and 24 patients (77.4\%) were females. The baseline patient characteristics are presented in Table 1 and Table 2.

All 35 aneurysms were of the saccular type : six (17.1\%) were located in the posterior circulation (basilar artery, five; superior cerebellar artery, one), while 29 (82.9\%) were located in the anterior circulation (anterior cerebral artery, nine; middle cerebral artery, one; internal carotid artery, 19). The mean aneurysm size was $5.2 \pm 2.6 \mathrm{~mm}$ (range, 2.011.8). Of the 35 aneurysms, 34 (97.1\%) were wide-neck aneurysms, defined as aneurysms showing dome-neck ratio (D/N ratio) $\leq 2$ or neck size $\geq 4 \mathrm{~mm}$. The mean neck size was $3.5 \pm 1.0 \mathrm{~mm}$ (range, $1.5-5.2$ ).

\section{Procedural information and angiographic out- comes}

In all the patients and for all the aneurysms, the NeuroForm Atlas reached the target vessel and was successfully deployed at the neck of the aneurysm. All the stents were deployed as per preoperative planning in all the 22 unruptured cases. All coiling procedures were performed using a jailing technique; although, a trans-strut technique was used for additional coil-

Table 2. Ruptured aneurysm features and details of the angiographic procedure

\begin{tabular}{|c|c|c|c|c|c|c|c|c|c|}
\hline No. & Location & $\begin{array}{c}\text { Parent } \\
\text { vessel } \\
\text { diameter } \\
\text { proximal } \\
(\mathrm{mm})\end{array}$ & $\begin{array}{c}\text { Parent } \\
\text { vessel } \\
\text { diameter } \\
\text { distal }(\mathrm{mm})\end{array}$ & $\begin{array}{c}\text { Dome/neck } \\
(\mathrm{mm})\end{array}$ & Treatment & Stent size & RROC & $\begin{array}{l}\text { Clinical } \\
\text { outcomes } \\
\text { BP/AD }\end{array}$ & Etc. \\
\hline 23 & $\mathrm{ACOA}$ & 3.3 & 2.4 & $6.6 / 4.6$ & $d_{1} j$ & $3.0 \times 15$ & 2 & $4 / 1$ & \\
\hline 24 & BA & 3.5 & 1.9 & $3.0 / 4.3$ & j & $3.0 \times 21$ & 1 & $5 / 6$ & Rebleeding \\
\hline 25 & ICA dorsal wall & 4.4 & 4.3 & $1.2 / 2.0$ & $j, s$ & $4.5 \times 21$ & 1 & $4 / 4$ & \\
\hline 26 & PCOA & 5.6 & 4.7 & $11.0 / 6.0$ & $d_{1} j$ & $4.5 \times 21$ & 1 & $5 / 4$ & \\
\hline 27 & $\mathrm{ACOA}$ & 3.0 & 2.9 & $3.5 / 4.0$ & $d, j$ & $3.0 \times 15$ & 1 & $5 / 2$ & \\
\hline 28 & $\mathrm{ACOA}$ & 2.2 & 2.2 & $10.3 / 4.8$ & $d, j$ & $3.0 \times 15$ & 2 & $5 / 4$ & \\
\hline 29 & PCoA & 4.7 & 3.7 & $11.5 / 4.7$ & j & $4.5 \times 21$ & 1 & $3 / 3$ & \\
\hline 30 & PCOA & 5.5 & 4.1 & $13.9 / 6.2$ & $d_{1} j$ & $4.5 \times 21$ & 1 & $4 / 1$ & Last coil migration \\
\hline 31 & $\mathrm{ACOA}$ & 3.3 & 2.8 & $3.2 / 2.2$ & j & $3.0 \times 15$ & 1 & $3 / 0$ & \\
\hline 32 & $A 2-3$ & 2.6 & 2.0 & $2.5 / 2.0$ & $j, s$ & $3.0 \times 15$ & 1 & $4 / 6$ & $\begin{array}{l}\text { DKA-hypoxic brain } \\
\text { damage }\end{array}$ \\
\hline 33 & ICA dorsal wall & 4.7 & 3.0 & $2.5 / 3.0$ & s & $4.5 \times 21$ & 2 & $4 / 2$ & \\
\hline
\end{tabular}

RROC : Raymond-Roy occlusion classification, BP : before procedure, AD : after discharge, ACoA : anterior communicating artery, $d$ : double catheter technique, $\mathrm{j}$ : jailing technique, BA : basilar artery, ICA : internal carotid artery, s : trans-strut technique, PCoA : posterior communicating artery, DKA : diabetic ketoacidosis 
ing in three cases without complications. In 21/22 (95.4\%) cases, the stent was deployed at the appropriate site. However, in one case, stent was deployed at an unexpected location because the microcatheter moved during opening for stent deployment (Fig. 1). Immediate, complete occlusion was evident in 16 of 24 aneurysms (66.7\%), while neck remnants persisted in six patients (25\%). Residual aneurysms were evident in two patients (8.3\%).

Among 11 patients with ruptured aneurysms, five underwent planned stent-assisted embolization. In six patients, stents were used to overcome unexpected events including thromboembolic complications (Fig. 2), final coil protrusion (Fig. 3) and coil instability (Fig. 4), and thromboembolic complications. Immediate angiographic revealed that complete occlusion occurred in eight patients (RROC I), while residual neck remnants were observed in the three patients (18.1\%).

In one case (3.3\%, case No. 30), intraarterial thrombolysis was required to address an immediate in-stent thrombus, which occurred during the procedure and was resolved without any associated complications.

\section{Clinical outcomes}

Patients with unruptured aneurysms showed no neurologic deficits prior to the procedure and were discharged without any clinical complications related to the procedure. Among the 11 patients with ruptured aneurysms, two died within a week after the procedure. One patient died due to hypoxic brain damage associated with re-bleeding just prior to the procedure, while the other died due to diabetic ketoacidosis caused by uncontrolled glucose levels. At discharge, four of nine patients exhibited unfavorable outcomes related to subarachnoid hemorrhage (SAH) $(\mathrm{mRS}=4$ in three patients, $\mathrm{mRS}=3$ in one patient). All four patients experienced acute hydrocephalus with intraventricular hemorrhage and underwent extra-ventricular drainage as initial treatment. Two of these patients underwent ventriculoperitoneal shunt surgery. Fortunately, there were no instances of vasospasm, ipsilateral stroke, late thromboembolic events, or late hydrocephalus in other patients with ruptured aneurysms. Overall mortality and morbidity rates in our study were $6.5 \%(2 / 31)$ and $12.9 \%$ (3/31), respectively.

\section{Angiographic and clinical outcomes at 6 months}

Follow-up DSA results were available for 21 patients with unruptured aneurysms. Follow-up DSA was not performed in one patient due to pregnancy. Among the 23 aneurysms analyzed, complete obliteration was observed in 16 cases (69.5\%), while neck remnants were observed in six cases (26.1\%). One patient $(4.3 \%)$ had a residual aneurysm. Six patients with ruptured aneurysms underwent follow-up DSA at 6 months, excluding two patients that had died and three with unfavorable outcomes. Complete aneurysm obliteration was observed in
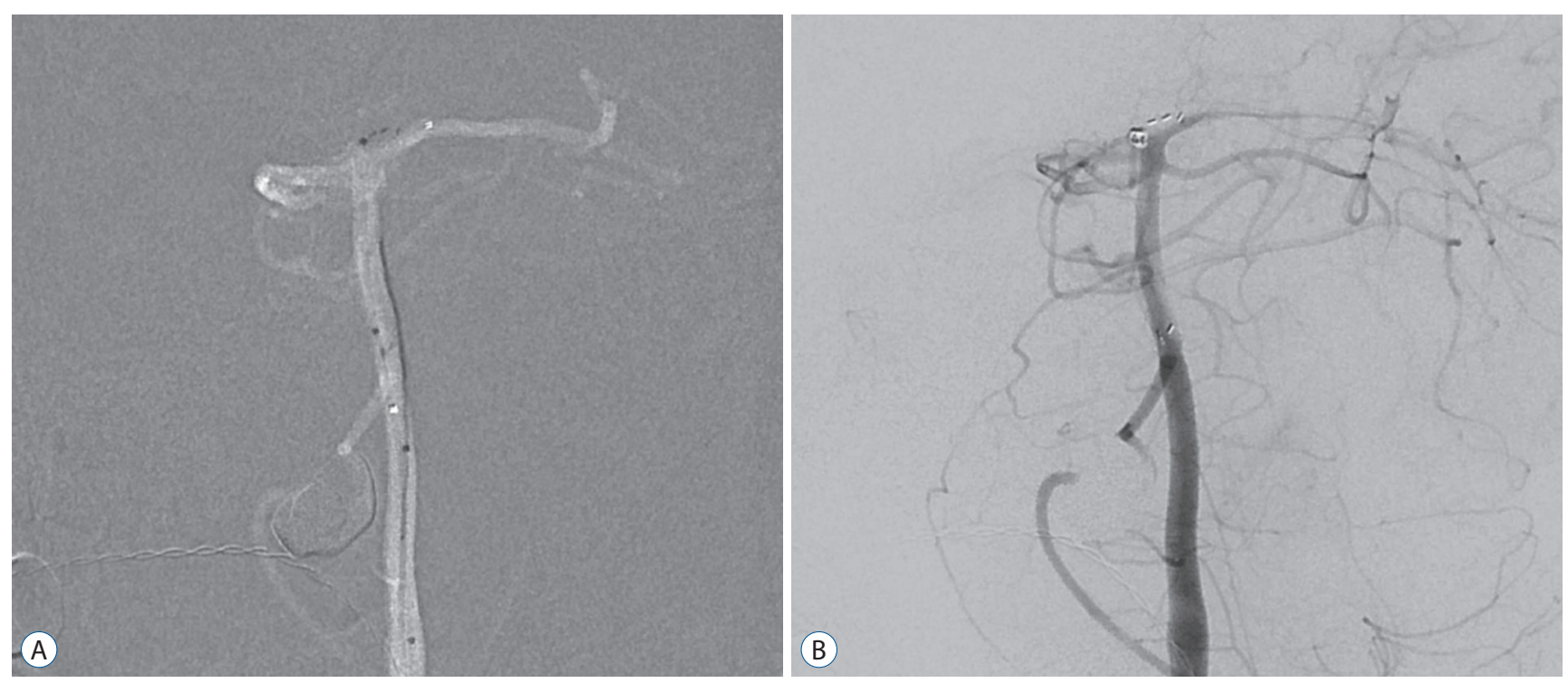

Fig. 1. Case 20. In the roadmap image, the stent was deployed at an inappropriate location. The stent was unable to completely cover the aneurysm neck (A). Due to an inappropriate stent position, the aneurysm was not completely occluded (B). 
three patients (50\%), while two patients (33.3\%) showed neck remnants. A residual aneurysm and growth of the aneurysmal sac were observed in one patient (16.7\%). Therefore, among the 29 aneurysms for which follow-up data were obtained, complete aneurysm obliteration was achieved in $65.5 \%$ (19/29), while $27.6 \%(8 / 29)$ showed neck remnants, and $6.9 \%$
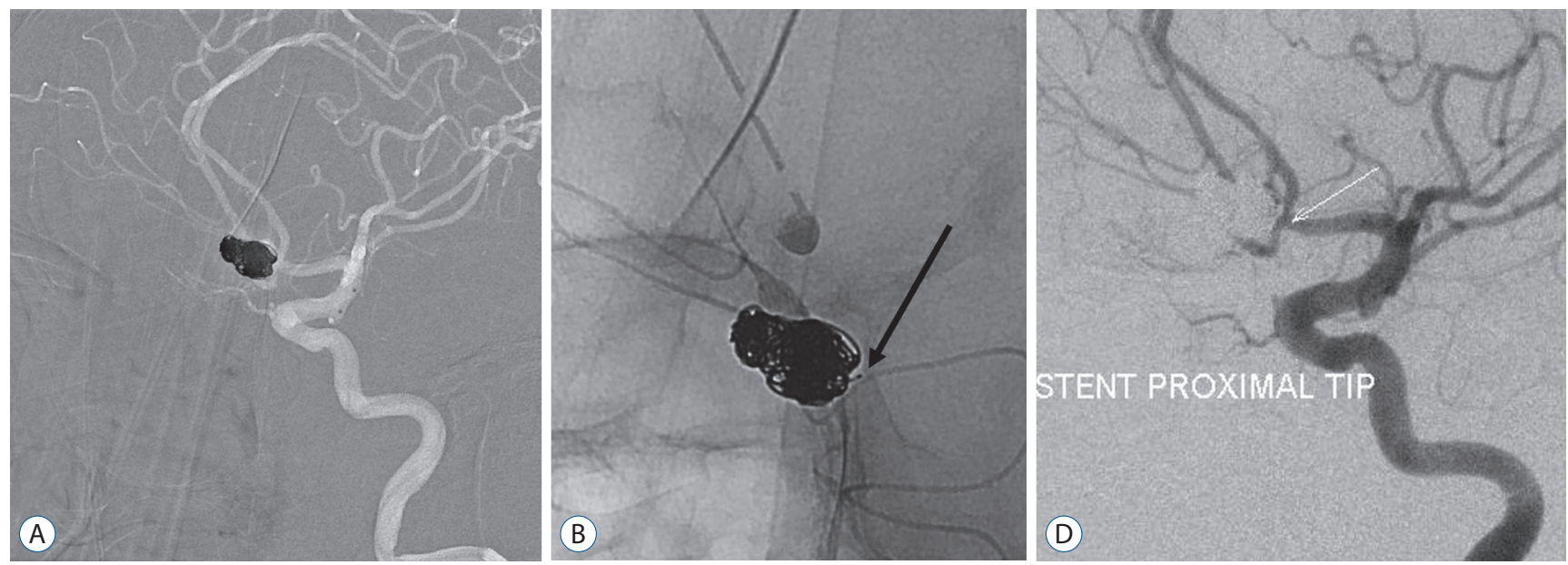

Fig. 2. Case 28. Ruptured anterior communicating artery aneurysm was successfully treated with the double catheter technique (A). However, the detachment zone (black arrow) of the last coil protruded to the parent artery (B), contralateral A2 flow was decreased/blocked due to the thrombus. The detachment zone of the protruding coil was inserted into the aneurysm using the NeuroForm Atlas stent (white arrow) and the flow was restored (C).
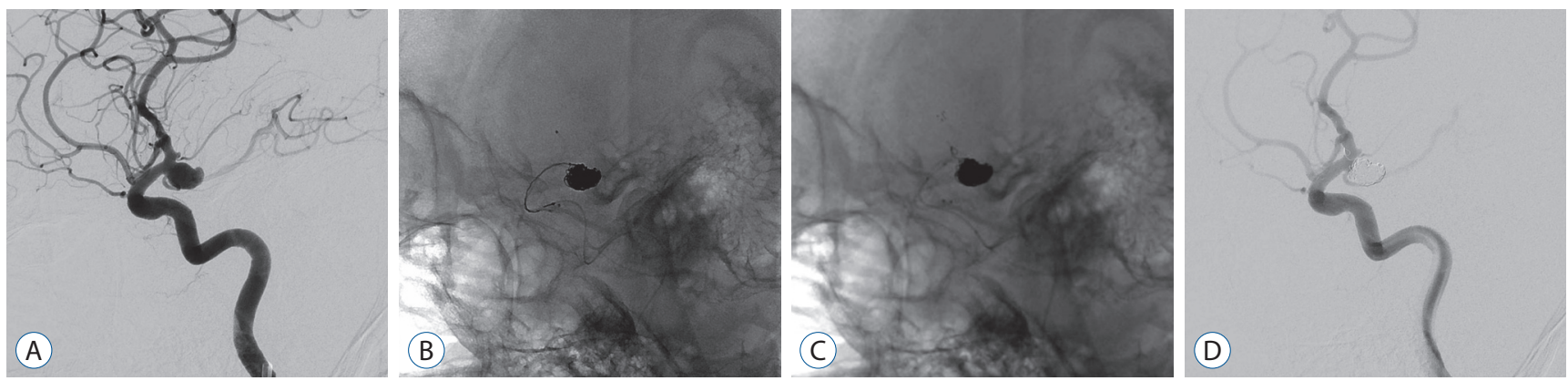

Fig. 3. Case 30. A patient with a ruptured posterior communicating artery aneurysm (A). A double catheter technique was used to treat the aneurysm and if the coil was protruded, a microcatheter was used to deploy the stent (B). The last coil protruded into the parent artery (C), an unstable migrating coil loop addressed via deployment of the NeuroForm Atlas stent (D).


Fig. 4. Case 33. Stent-assisted coil embolization of the ruptured internal carotid artery dorsal wall aneurysm was performed (A). In the follow-up angiogram after 3 weeks, contrast filling of the sac and movement of the protruding unstable coil loop were observed (B). Very severe vessel tortuosity was unavailable for sac selection, and an additional NeuroForm Atlas stent was deployed (C). The aneurysm sac contrast filling was not visible and the coil loop was fixed (D). 
(2/29) revealed residual aneurysms.

Patients with unruptured aneurysms exhibited no neurological symptoms at follow-up. Five of nine patients with ruptured aneurysms recovered without neurologic symptoms. Three patients with mRS scores of 4 were admitted to the hospital, while one patient with an mRS score of 3 was admitted to a rehabilitation facility. Thus, favorable outcomes were achieved in $86.2 \%$ of patients $(25 / 29)$ (mRS score $<2$ ).

\section{DISCUSSION}

In our study, we evaluated the safety and efficacy of the new NeuroForm Atlas microstent device used during coil embolization of wide-neck aneurysms. In our study, all stents were deployed successfully, and all aneurysms underwent coil embolization without technical problems. Several features of the NeuroForm Atlas stent help in successful deployment. The stent can be delivered via a 0.0165 inch or 0.017 inch microcatheter, the size used for coil delivery. This makes obtaining access to the distal aneurysms with small parent arteries easier. In addition, The NeuroForm Atlas stent has a unique structure that utilizes a closed cell for re-crossing of the microcatheter and an open cell for better anchorage and wall apposition ${ }^{6}$. These changes were introduced to improve stent deliverability, vessel wall apposition, and coil scaffolding and to overcome the foreshortening problems associated with the NeuroForm EZ system. Furthermore, smaller and increased numbers of alternative open cells provide better scaffolding and allow adequate coil stability at the bifurcated aneurysm or highly tortuous parent arteries. In addition, when compared to the braded stent, the low metal-to-artery ratio $(6-12 \%)$ and segmental expansion ability of the stent system result in highly successful stent deployment without changing the parent artery direction. In addition, the rate of foreshortening of the NeuroForm Atlas stent is very low (2.9-6.3\%), enabling very high deployment accuracy. Due to these advantages, successful deployments are similar to or better than those reported with the use of other stent systems such as the LVIS Jr. microstent, with which Behme et al. reported a success rate of $100 \%$, or the LEO Baby stent, with which Aydin et al. reported a success rate of $93.5 \%{ }^{1,2}$.

In our study, no cases of stent-related ipsilateral stroke or neurological death occurred at the 6-month follow-up, result- ing in a relative safety of $100 \%$. Moreover, favorable outcomes were observed in $86.2 \%$ patients $(25 / 29)$. This result is in accordance with those of Ten Brinck et al. ${ }^{10)}$, who reported favorable outcomes in $84.6 \%$ patients (22/26) treated using the NeuroForm Atlas stent in a preliminary study. Although only two patients with SAH (2/27) were included, Ulfert et al. ${ }^{11)}$ reported that none of their patients developed new symptoms or experienced permanent reductions in mRS scores. Another study by Jankowitz et al. ${ }^{6)}$ reporting the safety of NeuroForm Atlas system documented a stent-associated late thromboembolic event in one of the 30 patients with unruptured aneurysms (3.3\%) at the 12-month follow-up. Taken together, these results suggest that the NeuroForm Atlas microstent exhibits comparable safety to the LVIS Jr. system²).

In our study, immediate favorable outcomes and complete occlusion were observed in 94.2\% (33/35) and 68.5\% (24/35) of cases, respectively. At the 6-month follow-up, successful occlusion was observed in 93.1\% (27/29), and complete occlusion was observed in $65.5 \%$ (16/29) of cases. Ten Brinck et al. ${ }^{10)}$ reported favorable and complete occlusion rates of $69.2 \%$ and $53.8 \%$ at 6 months, respectively. In addition, Ulfert et al. ${ }^{11)}$ reported satisfactory immediate angiographic outcomes: complete aneurysm obliteration was observed in $84 \%$ cases, while neck remnants were observed in the remaining $16 \%$. Furthermore, high-resolution time-of-flight magnetic resonance angiography revealed an occlusion rate of $100 \%$ at the 5-month follow-up ${ }^{11)}$. Jankowitz et al. ${ }^{6}$ reported a complete occlusion rate of $86.7 \%$ for the NeuroForm Atlas system. Follow-up occlusion rates for stent-assisted coil embolization using the LEO Baby or the LVIS Jr. systems range from $56.5 \%$ to $90.0 \%$, with one meta-analysis reporting a complete aneurysm obliteration rate of $60.6 \%{ }^{1,9,12}$. Our findings are in accordance with the results of these previous studies, highlighting the efficacy of the NeuroForm Atlas microstent.

Despite the structural advantages, the NeuroForm Atlas stents also have some drawbacks in use. When deploying the stent at a concave surface, it is difficult to re-access the aneurysmal sac due to the tightness of the open cells. In contrast to the woven-design stent, NeuroForm Atlas stent system cannot ensure flow diversion. In addition, the NeuroForm Atlas stent cannot be re-sheathed. Indeed, currently no method exists for dealing with stents that have been deployed at an inappropriate position, and excessive attempts at removal can affect coil stability and damage the intima of the parent artery. 
In the present study, case No. 20 was rather difficult, as the NeuroForm Atlas stent could not be re-sheathed. When the microstent is advanced through a relatively tortuous segment in the microcatheter, the microcatheter has a tendency for spontaneous withdrawal. To address this issue, a closed-cell stent can be re-introduced into the sheath, allowing for repositioning of the microcatheter and redeployment of the stent. However, as re-sheathing of open-cell stents such as the NeuroForm Atlas is not possible, surgeons must be aware of microcatheter tension and should carefully withdraw the microcatheter when the parent artery is tortuous. If the microcatheter is withdrawn before the distal tip of the stent exits the microcatheter, the transcatheter technique can be used. In this situation, the microcatheter containing the stent can be removed, and a new microcatheter can be securely positioned in the parent artery. This method may aid in reducing the risk of microcatheter drop and stent deployment at an undesired location.

The present study possesses some limitations of note, including its single-center retrospective design and short-term follow-up period. As our non-randomized study included only 31 consecutive patients, our results may have been influenced by selection bias. Furthermore, we did not include a control group of patients undergoing alternative endovascular treatments or patients with giant aneurysms. Indeed, only three patients had aneurysms larger than $10 \mathrm{~mm}$, while seven patients had aneurysms smaller than $3 \mathrm{~mm}$. However, our study is advantageous in that we utilized DSA at follow-up assessments.

\section{CONCLUSION}

In this study, we observed that stent-assisted coil embolization with NeuroForm Atlas stent may be safe and effective in the treatment of wide-neck intracranial aneurysms. Furthermore, NeuroForm Atlas stent-assisted coil embolization is feasible for the treatment of both ruptured and unruptured wide-neck aneurysms. However, long-term angiographic follow-up is required to completely elucidate the durability of the NeuroForm Atlas stent, as well as the associated recurrence and retreatment rates.

\section{CONFLICTS OF INTEREST}

No potential conflict of interest relevant to this article was reported.

\section{INFORMED CONSENT}

This type of study does not require informed consent.

\section{AUTHOR CONTRIBUTIONS}

\author{
Conceptualization : SWL \\ Data curation : CHK, YHK \\ Formal analysis : SKS \\ Funding acquisition : $\mathrm{CHK}$ \\ Methodology : DWS \\ Project administration : SWL, GSS \\ Visualization : YHK \\ Writing - original draft : $\mathrm{CHK}$ \\ Writing - review \& editing : SWL
}

\section{- Acknowledgements}

This study was supported by Research institute for Convergence of biomedical science and technology (30-2017-009), Pusan National University Yangsan Hospital.

\section{References}

1. Aydin K, Arat A, Sencer S, Barburoglu M, Men S : Stent-assisted coiling of wide-neck intracranial aneurysms using low-profile LEO baby stents: initial and midterm results. AJNR Am J Neuroradiol 36 : 1934-1941, 2015

2. Behme D, Weber A, Kowoll A, Berlis A, Burke TH, Weber W : Lowprofile visualized intraluminal support device (LVIS Jr) as a novel tool in the treatment of wide-necked intracranial aneurysms: Initial experience in 32 cases. J Neurointerv Surg 7 : 281-285, 2015

3. Brilstra EH, Rinkel GJ, van der Graaf Y, van Rooij WJ, Algra A : Treatment of intracranial aneurysms by embolization with coils: a systematic review. Stroke 30 : 470-476, 1999

4. Cho WS, Kim JE, Park SQ, Ko JK, Kim DW, Park JC, et al. : Korean clinical practice guidelines for aneurysmal subarachnoid hemorrhage. J Korean Neurosurg Soc 61 : 127-166, 2018 
5. Ge H, Lv $X$, Yang $X$, He H, Jin $H$, Li Y : Lvis stent versus enterprise stent for the treatment of unruptured intracranial aneurysms. World Neurosurg $91: 365-370,2016$

6. Jankowitz BT, Hanel R, Jadhav AP, Loy DN, Frei D, Siddiqui AH, et al. : Neuroform Atlas stent system for the treatment of intracranial aneurysm: primary results of the atlas humanitarian device exemption cohort. J Neurointerv Surg $11: 801-806,2019$

7. King B, Vaziri S, Singla A, Fargen KM, Mocco J : Clinical and angiographic outcomes after stent-assisted coiling of cerebral aneurysms with enterprise and neuroform stents: a comparative analysis of the literature. J Neurointerv Surg 7 : 905-909, 2015

8. Molyneux AJ, Birks J, Clarke A, Sneade M, Kerr RS : The durability of endovascular coiling versus neurosurgical clipping of ruptured cerebral aneurysms: 18 year follow-up of the UK cohort of the international subarachnoid aneurysm trial (ISAT). Lancet 385 : 691-697, 2015

9. Poncyljusz W, Bilinski P, Safranow K, Baron J, Zbroszczyk M, Jaworski M, et al. : The LVIS/LVIS Jr. stents in the treatment of wide-neck intracranial aneurysms: multicentre registry. J Neurointerv Surg 7 : 524-529, 2015

10. Ten Brinck MFM, de Vries J, Bartels RHMA, Grotenhuis JA, Boogaarts $H D$ : NeuroForm Atlas stent-assisted coiling: preliminary results. Neurosurgery 84 : 179-189, 2019

11. Ulfert C, Pham M, Sonnberger M, Amaya F, Trenkler J, Bendszus M, et al. : The Neuroform Atlas stent to assist coil embolization of intracranial aneurysms: a multicentre experience. J Neurointerv Surg 10 : 11921196, 2018

12. Wang F, Chen X, Wang Y, Bai P, Wang HZ, Sun T, et al. : Stent-assisted coiling and balloon-assisted coiling in the management of intracranial aneurysms: a systematic review \& meta-analysis. J Neurol Sci 364 : 160-166, 2016

13. Yoon CH, Lee HW, Kim YS, Lee SW, Yeom JA, Roh J, et al. : Preliminary study of tirofiban infusion in coil embolization of ruptured intracranial aneurysms. Neurosurgery $82: 76-84,2018$ 